OPEN ACCESS

Edited by:

Sonnet Jonker

Oregon Health \& Science University,

United States

Reviewed by:

Ivan Gomez-Mestre,

Estación Biológica de Doñana (EBD),

Spain

Kent L. Thornburg,

Oregon Health \& Science University,

United States

*Correspondence:

Warren W. Burggren

burggren@unt.edu

Specialty section:

This article was submitted to Embryonic and Developmental

Physiology,

a section of the journal

Frontiers in Physiology

Received: 16 September 2019

Accepted: 27 December 2019

Published: 22 January 2020

Citation:

Burggren WW (2020) Phenotypic

Switching Resulting From

Developmental Plasticity: Fixed or

Reversible? Front. Physiol. 10:1634.

doi: 10.3389/fphys.2019.01634

\section{Phenotypic Switching Resulting From Developmental Plasticity: Fixed or Reversible?}

\author{
Warren W. Burggren*
}

Developmental Integrative Biology, Department of Biological Sciences, University of North Texas, Denton, TX, United States

The prevalent view of developmental phenotypic switching holds that phenotype modifications occurring during critical windows of development are "irreversible" that is, once produced by environmental perturbation, the consequent juvenile and/or adult phenotypes are indelibly modified. Certainly, many such changes appear to be non-reversible later in life. Yet, whether animals with switched phenotypes during early development are unable to return to a normal range of adult phenotypes, or whether they do not experience the specific environmental conditions necessary for them to switch back to the normal range of adult phenotypes, remains an open question. Moreover, developmental critical windows are typically brief, early periods punctuating a much longer period of overall development. This leaves open additional developmental time for reversal (correction) of a switched phenotype resulting from an adverse environment early in development. Such reversal could occur from right after the critical window "closes," all the way into adulthood. In fact, examples abound of the capacity to return to normal adult phenotypes following phenotypic changes enabled by earlier developmental plasticity. Such examples include cold tolerance in the fruit fly, developmental switching of mouth formation in a nematode, organization of the spinal cord of larval zebrafish, camouflage pigmentation formation in larval newts, respiratory chemosensitivity in frogs, temperature-metabolism relations in turtles, development of vascular smooth muscle and kidney tissue in mammals, hatching/birth weight in numerous vertebrates,. More extreme cases of actual reversal (not just correction) occur in invertebrates (e.g., hydrozoans, barnacles) that actually 'backtrack' along normal developmental trajectories from adults back to earlier developmental stages. While developmental phenotypic switching is often viewed as a permanent deviation from the normal range of developmental plans, the concept of developmental phenotypic switching should be expanded to include sufficient plasticity allowing subsequent correction resulting in the normal adult phenotype.

Keywords: development, plasticity, evolution, environment, critical window

\section{DEVELOPMENT, PLASTICITY AND PHENOTYPE}

That developing animals are shaped by their environment is not a new concept. Aristotle deserves first credit for recognizing that animals will develop different forms when raised in different environments (Aristotle, 350BC). Of course we now frame these observations as changes in gene expression influenced by environmental stressors. In this essay I first briefly expand upon the 
Gene X Environment context to include development, epigenetics and other factors, and then consider whether the product of these interactions (e.g., modified phenotypes enabled by developmental plasticity) are fixed or reversible.

\section{Moving Beyond the Gene-Environment Interaction Paradigm in the Context of Development}

Phenotypic plasticity is a venerable, long-standing concept, and is often framed as the interactions of genes $(G)$ by environment (E), creating a specific phenotype that is the result of socalled ' $G \times E$ ' interactions. This concept has been reviewed dozens of times as both a general concept as well as in specific contexts such as human pathologies and disease - an entry into the voluminous literature can be gained from Moffitt et al. (2005), Manuck and McCaffery (2014), Halldorsdottir and Binder (2017), Josephs (2018), and Saltz et al. (2018). Much of the discussion of environmentally induced changes in gene expression resulting in modified phenotype does not explicitly involve a developmental component, though certainly much is known about developmental phenotypic plasticity in both plants and animals - for reviews see West-Eberhard (2005), Spicer and Rundle (2007), Uller (2008), de Jong and Leyser (2012), Bateson et al. (2014), Standen et al. (2014), Burggren and Mueller (2015), Bateson (2017), and Burggren (2018). Thus, for developing animals, the classic $\mathrm{G} \times \mathrm{E}$ framework is expanded to the more encompassing (Genes $\times$ Environment $) \times$ Development, or $(G \times E) \times D$. Note that it has been suggested that Time $(T)$ and even allometry be a component of this construct (Pigliucci et al., 1996), but in this essay we consider development as a distinct sub-set of time (Dubansky, 2018).

It is becoming increasingly clear that, to this basic formula describing developmental phenotype, we must add an epigenetic component ('Epi') representing intragenerational and especially intergenerational environmental experiences resulting in modified gene expression (Skinner, 2011; Jablonka, 2013; Burggren and Crews, 2014; Skinner, 2015; Burggren, 2016, 2017). The influences of epigenetic readers, writers and erasers on the epigenetic markers that influence gene expression can result from changes ranging from minutes to across multiple generations. Thus, the formula $\mathrm{G} \times \mathrm{E} \times \mathrm{D}$, which assumes that gene expression results from contemporaneous environmental conditions, can be expanded to $\mathrm{G} \times(\mathrm{E}+\mathrm{Epi}) \times \mathrm{D}$. This new expression now includes both within-life altered gene expression patterns as well as both known and unknown epigenetic factors from past parental/ancestral experiences through altered gene expression patterns in germ cells. These epigenetic changes, combined with ambient environmental affects on gene expression, contribute to the control of the phenotype that emerges during development. Importantly, these genomic/epigenomic interactions form the basis for the differing phenotypic outcomes that lead to successful survival in stressful environments.

Finally, an additional source of phenotype switching has been proposed to emerge from stochastically driven cell differentiation during early development that is amplified as tissue differentiation and growth progresses (Woods, 2014). Developing animals might then be a mosaic of both programed and stochastic development. As a consequence, the originally lauded but now limiting ' $G \times E$ ' construct could be expanded to $G \times(E+E p i) \times(D \times S)$, where $S$ represents the influence of stochasticity. In suggesting this, it is realized that there will doubtlessly be disagreement as to where the brackets are placed in this 'formula', whether the $\times$ symbols should in fact be + signs, etc. etc. The point of this or any other next-generation formulae encompassing these concepts is that the $\mathrm{G} \times \mathrm{E}$ paradigm is now more properly regarded as an overly simplistic, historical view of how animals interact with their environment during development and maturation.

All of these factors - genes, environment, development, epigentic markers and stochastic changes in the developmental plan - interact together in complex and sometimes unpredictable ways, and lead to modified phenotypes resulting from developmental phenotypic plasticity. Importantly, however, there is also a crucial element of timing, in that the expression of genes, appearance of stressors in the environment, progression of development, and the writing and erasing of epigenetic markers all march to the beat of a different drummer. No where is this more evident than in the concept of "critical windows" during development, which will now be explored.

\section{Critical Windows, Developmental Plasticity, and Phenotype Switching}

To heavily paraphrase T. Dobzhansky's famous phrase on biology and evolution (Dobzhansky, 1973), nothing in development makes sense except in the light of critical windows (also known as sensitive periods). The terms 'critical moment' and 'sensitive period' were first used in the early 20th century by the influential embryologist Charles Stockard, who showed that oxygen deprivation during certain specific periods of development created developmental anomalies in Fundulus, trout and frogs (Stockard, 1921). These observations helped explain the relationship between time in development and susceptibility to environmental influences, a relationship further explored in Nobel laureate Hans Spemann's Embryonic Development and Induction (Spemann, 1938). Likely drawing upon the analogy with biology, in the late 1950s neurologists Wilder Penfield and Lamar Roberts advanced the "critical period hypothesis" related to neural development and language acquisition (Penfield and Roberts, 1959). Lenneberg (1967) further promoted this notion for human development in his book Biological Foundations of Language. Noteworthy is that, somewhat in contrast to the embryological literature that focuses on maladaptations (usually morphological) emerging during critical windows, in the human linguistics and psychological literature, the critical window is often regarded as a period when certain environmental exposures are actually necessary and important for language development and other aspects of normal development, e.g., Hensch and Bilimoria (2012). 
Currently, investigators typically describe a critical window as a specific, defined period during development when $\mathrm{G} \times \mathrm{E} \times \mathrm{D}$ (and, studied less often, $[\mathrm{G} \times(\mathrm{E}+\mathrm{Epi}) \times \mathrm{D}]$ interactions result in subsequently switched (modified) phenotypes that differ from the normally expected range of developmental trajectory. Numerous authors have offered up similar definitions - e.g., Kunes and Zicha (2006), Ferner and Mortola (2009), Ali et al. (2011), Burggren and Reyna (2011), Vickers (2011), Voss (2013), Alvine and Burggren (2014), Burggren et al. (2014), Burggren and Mueller (2015), Eme et al. (2015), and Pelster and Burggren (2018).

The concept of critical window is the subject of considerable investigation in disciplines ranging from physiology to ecology to toxicology. Indeed, at the time of the writing of this article, the Pubmed data base listed $>4400$ articles evoking critical windows (the majority being in animal rather than plant development), and $>32,000$ articles describing sensitive periods in a human psychological context. While there is huge variation in observations and experiments on critical windows and their effects, Table 1 outlines some of the key general characteristics of critical windows, along with some examples of resulting phenotype switching.

\section{ARE CHANGES THAT ARE EVOKED DURING CRITICAL WINDOWS REVERSIBLE OR FIXED?}

\section{Definitions and Semantics}

Most organisms typically have at least some capacity to counteract potentially negative effects of environmental fluctuations that they experienced during development or even later in life as adults. Yet, a commonly (though not universally) posited key characteristic of developmental phenotypic plasticity is that a switched phenotype produced specifically as a result of developmental plasticity is essentially irreversible - e.g., Wilson and Franklin (2002); West-Eberhard (2003), Matesanz et al. (2010, 2012), Utz et al. (2014), Woods (2014), Senner et al. (2015), Beaman et al. (2016), Slotsbo et al. (2016), and Noh et al. (2017). This view is especially held to be true when prevalent when phenotypic switching occurs during a narrow developmental window. Interestingly, Woods (2014) employs the term phenotypic flexibility for reversible (or additional new) phenotypic changes. Utz et al. (2014) uses the term reversible if a trait's phenotype "...can be reversed to the original state." Some authors do not distinguish between 'plasticity' and 'flexibility' or even see the need to, whereas others painstakingly lay out their ground for the irreversibility of phenotypic modification during development - for discussion, see Debat and David (2001), Gabriel (2005), Woods (2014), and Sommer et al. (2017). Investigators studying cold hardening in developing insects have differentiated between irreversible "developmental acclimation" and reversible "short-term acclimation" (Noh et al., 2017). Finally, several studies have differentiated between 'developmental' and 'reversible' environmentally induced phenotypic modification (Angiletta, 2009; Munday et al., 2013; Utz et al., 2014; Donelson et al., 2017).

Regarding definitions, it is not the intention of this review to add to the existing definitional morass surrounding the definition of developmental phenotypic plasticity and resultant phenotypic switching ${ }^{1}$ (Korzybski, 1933). Yet, perhaps the additional term 'correctable' should be inserted into the discussion of terms. Why? Correctable indicates that the normal range of adult phenotypes could still be achieved despite potentially transient phenotypic modification during development - that is, the switched phenotype can be corrected to the phenotype that would result if gene expression had not been modified by environmental or epigenetic influences. This would leave the term 'reversible' to apply to the unusual phenomenon of a true reversal (backtracking) along a developmental pathway (which can indeed occur, as discussed below). Yet, the problem with this reversible/correctable semantic conundrum is that the term 'correctable' suggests that the modified phenotype resulting from developmental plasticity is actually sub-optimal (i.e., needs to be 'corrected'). In fact, the modified phenotype emerging from (enabled by) developmental plasticity might, of course, actually be advantageous under current environmental conditions - e.g., Toth and Hettyey (2018); Bautista and Burggren (2019), and Mendez-Sanchez and Burggren (2019). Not being able to resolve this issue here, this essay will nonetheless use the commonly acceptable term reversible with a parenthetical inclusion of correctable to remind the reader of the complexity of developmental plasticity.

\section{We Find What We Look For}

Importantly, the prevailing view that phenotypic switching during critical windows of development is irreversible, likely emerges from a truth buried within developmental dogma. In fact, many of the described examples of phenotypic switching induced during an often-narrow critical window for a trait indeed appear to be irreversible (Burggren and Reyna, 2011; Daskalakis et al., 2013; Burggren and Mueller, 2015; Senner et al., 2015; Lloyd and Saglani, 2017). Moreover, stark examples such as the production of 2-headed frogs induced by hypoxia experienced during a critical window (Stockard, 1921) certainly contribute to the notion that switched phenotypes cannot be reversed. Yet, developmental critical windows are typically quite brief periods during a much longer period of overall development, and these extended developmental periods can potentially allow sufficient time for the animal to regain some or all of its normal phenotype.

\footnotetext{
${ }^{1}$ The philosopher Popper (1970) suggested that “...we should altogether avoid, like the plague, discussing the meaning of words." While one can be sympathetic to his viewpoint, due diligence requires acknowledging differing definitions in the long-standing debate as to the reversibility of effects resulting from developmental plasticity. Underscoring this point, the two reviewers of this manuscript had differing opinions on whether the field viewed phenotypic switching as reversible or irreversible when it occurred during development. Ultimately, perhaps Alfred Korzybski (1879-1950), the father of the field of General Semantics, got it right when he posited that "Whatever you say it is, it isn't" (Korzybski, 1933).
} 
TABLE 1 | Characteristics of critical windows for development.

\begin{tabular}{ll}
\hline $\begin{array}{l}\text { Characteristics of } \\
\text { critical windows }\end{array}$ & Description \\
\hline Timing in & Critical developmental windows can \\
Development of & occur from shortly after egg fertilization \\
Critical Windows & until achievement of sexual maturity.
\end{tabular}

Examples

Human psychiatric illnesses frequently first manifest during critical windows in teenagers, potentially associated with gut microbiome dysfunction.

Human cardiovascular form and function impacted by environmental toxicants during critical windows starting as early as week 2 after conception
Duration ('Width') of Critical Window
Finite "width" to critical window - i.e., distinct onset and closing of window, but interpretation of critical window "edges" is dependent upon stressor dose
Cardiac development in chicken embryos primarily sensitive during week 2 of 3 weeks incubation

Gonad differentiation in zebrafish between 30-44 days post-fertilization

Modeling of critical window as a 3D construct of time, dose and phenotype

\begin{tabular}{ll}
\hline Duration of Switched & $\begin{array}{l}\text { Phenotypic switching irreversible, } \\
\text { persisting through subsequent life } \\
\text { Phenotype }\end{array}$
\end{tabular}

Number of Critical

Windows Per Trait

Typically only one, but multiple critical windows can exist for same trait
Larval hypoxia has long-term effects on protein digestion and growth in juvenile European sea bass

Chicken embryos show aberrant aortic arch morphogenesis when hemodynamic variables are manipulated specifically at Stage 21 .
Lipid and glucose metabolism in adult sheep is similarly affected by undernutrition early in gestation as well as immediately postnatally

References

McVey Neufeld et al., 2016

Lage et al., 2012

Chan and Burggren, 2005

Quintaneiro et al., 2019

Burggren and Mueller, 2015

Zambonino-Infante

et al., 2017

Kowalski et al., 2013

Correction of structural abnormalities in mouse brain cortex have multiple critical windows

Poore et al., 2010

Cox et al., 2018

During Critical

Window

Organ System

Human populations differ in critical window for infant weight gain and its effect on adult adiposity

Hypersalinity delays onset of heartbeat and changes timing of foot attachment and eye spot formation in the euryhaline snail Radix balthica

Critical windows for nephrogenesis and morphologica renal development differ between dog, pig, rabbit, monkey, mouse, and rat

Critical windows for motor activity and motor function performance identified by exposure to environmental neurotoxins differ in rats and mice

Critical window for gut microbiome establishment differs between wood frogs, green frogs and bullfrogs
Odors (aversive or attractive) in first week post-eclosion fruit fly larvae alter olfactory circuitry

Hypoxia during middle third of avian incubation alters gross morphology and metabolic physiology

Stressors can be intrinsic or extrinisic (environmental) factors.
Phenotypic switching during critical windows is dose-dependent
Body mass changes in Artemia during early development are dependent on strength of environmental salinity

Hypoxia-induced alteractions of morphology and physiology of chicken embryo show differential responses to 13 and $15 \%$ oxygen

Sex Differences in

Critical Window

Susceptibility for

Same Trait
Phenotypic switching during critical windows is sex-dependent
Prenatal critical window for oranotin toxicant exposure in rats results in greater permanent phenotype switching in males compared to females
Prenatal critical window for particulate air pollution exposure causes Hsu et al., 2015 phenotype switching in human male but not female children

Critical window for hypoxic effects on heart mass and blood

Tate et al., 2015
Timing of development of window differs between organ systems within an organism Differences in Critical Windows

pressure are considerably different in timing and width in embryonic alligator hearts

Critical windows for sensitivity to environmental toxicant differ in timing and duration for immune and respiratory systems in humans

Population Differences in Critical Windows For Same Trait
Timing and width of critical window for a particular phenotypic trait varies "heterokairy" between different populations - i.e.,

Golovin and Broadie, 2016

Dzialowski et al., 2002

Mueller et al., 2016

Zhang and Burggren, 2012

Grote et al., 2009

Dietert et al., 2000

Wells, 2014

Tills et al., 2010

Frazier, 2017 phenotypic trait varies between different phenotypic trait varies between
species - i.e., "heterochrony"
Ingber and Pohl, 2016

(Ingber and Pohl, 2016) 


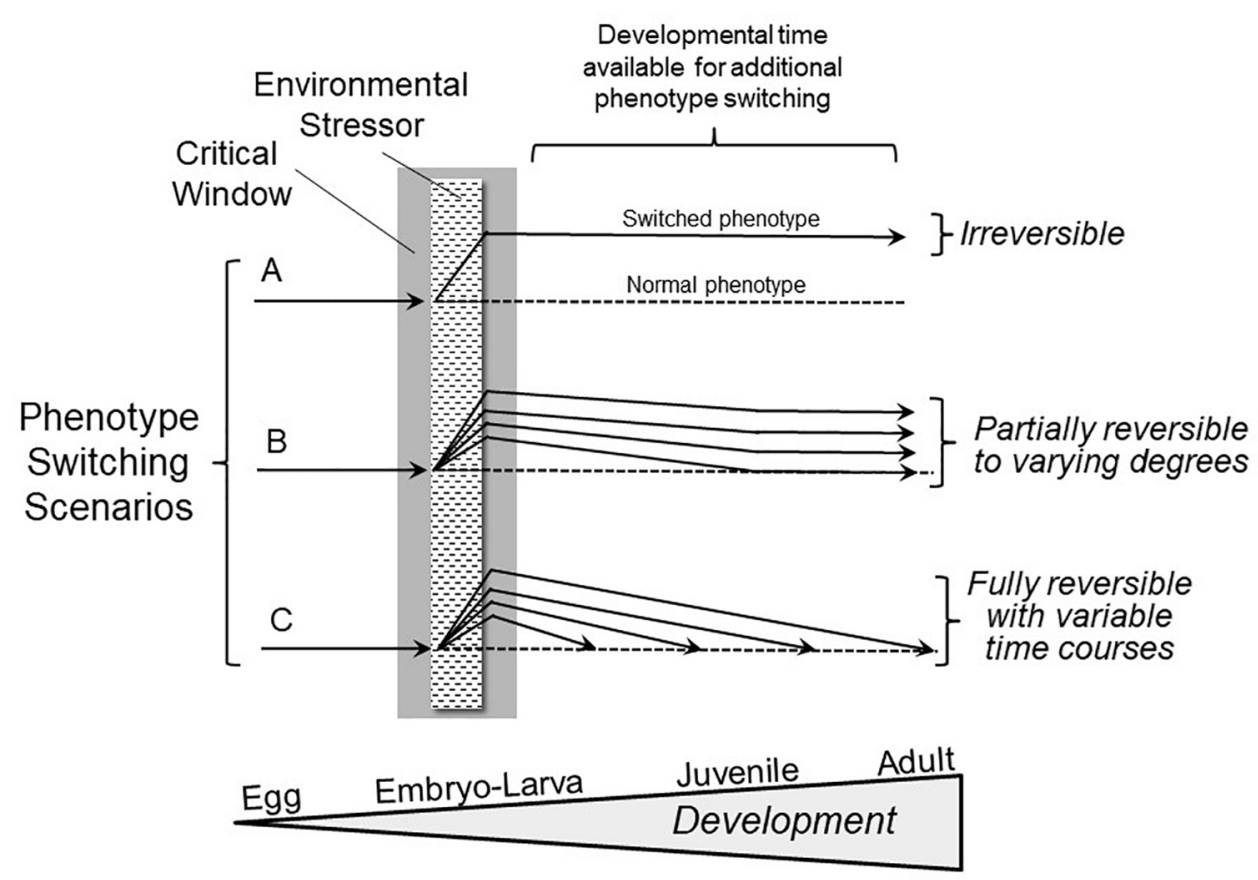

FIGURE 1 | Hypothetical outcomes involving phenotype switching scenarios following exposure to adverse environments during critical windows in development. Scenario A shows the conventional, highly discussed condition of irreversible switching of phenotype induced by an environmental stressor during the critical window. Scenario B depicts a far less frequently studied situation of phenotypic switching initiated during a critical window that, in fact, is reversible to varying degrees. Scenario C indicates that there may be complete reversibility of phenotypic modifications occurring at varying rates during subsequent development. The extent and rate of phenotype reversal may relate to the extent of the difference between the altered and normal (typical) juvenile/adult phenotypes. Importantly, either a single trait might show different degrees of reversibility, or multiple traits could show a range of degrees of reversal to the normal phenotype.

As Utz et al. (2014) comment, "Most theoretical investigations on plasticity are restricted to irreversible plasticity. i.e., the expression of a specific phenotype is determined during development and remains unchanged during the whole life of an organism...". As these authors go on to indicate, there are three categories of traits - non-plastic, irreversibly plastic, and reversibly plastic. An important point that Utz et al. (2014) make, and that I wish to underscore in this essay, is that most studies have focused on irreversible traits. Such traits are certainly in abundance, but do not represent the full picture of developmental phenotypic plasticity and phenotype switching. Consequently, a well populated database has been developed that is skewed toward non-reversible traits. Put differently, if one looks for irreversible phenotypic switching, one will most certainly find it. As a result, an overly restricted working definition of developmental phenotypic plasticity seems to have emerged in which the concept of reversibility has little or no traction. Against this backdrop, Lande (2014) advocates moving beyond previous theory that assumes a constant adult phenotype results from plasticity during development, to a more nuanced view that considers multiple factors. These include the cost of plasticity, the environmental variance and predictability, and developmental rates and characteristics (Lande, 2014).

Notably, an overly narrow definition has prevailed to date largely because we are not conducting experiments specifically designed to reveal reversible phenotype switching during development. Consider Hempel's and Popper's "Ravens Paradox," where finding a single white raven falsifies the hypothesis that all ravens are black (Hempel, 1945; Popper, 1959). Following their logic, we only need to find one example of a phenotypic modification occurring during a developmental critical window that can be subsequently reversed ("the white raven") to falsify the hypothesis that all phenotypic switching during development is irreversible ("all ravens are black"). If reversible phenotypic switching initially induced during the critical window were to occur, what would it actually look like? Figure 1 presents a broad view of the expression of developmental plasticity that includes reversible phenotypic switching. In the most highly studied scenario (Figure 1A) changes evoked by an environmental stressor are deemed irreversible, and as indicated earlier, there are certainly many examples of this phenomenon. However, Scenarios B and C provide for varying degrees and rates of reversibility of phenotypes initially produced as a result of stressors during the critical window.

It is important to emphasize that there are no theoretical or practical objections to reversal of phenotypic switching originating during a developmental critical windows. But, if the field of developmental biology chooses to define that particular subset of clearly non-reversible phenotypic changes occurring during development as, in fact, representing all 'developmental phenotypic plasticity', we exclude real phenomena that don't fit into that overly restrictive definition. Moreover, it means we would have to come up 
with yet another term to cover those examples of reversible (correctable) phenotypic switching that are induced during the critical window for development. Perhaps more efficient is simply convincing the reader that there are, indeed, clear examples of reversible phenotypic switching resulting from developmental phenotypic plasticity. Let us now consider the considerable body of evidence for reversible phenotypic plasticity.

\section{REVERSIBLE DEVELOPMENTAL PHENOTYPIC SWITCHING: THE EVIDENCE}

\section{Reversible (Correctable) Phenotypic Switching During Development}

In the most common pattern of reversible phenotypic switching, a developing organism takes a novel developmental trajectory in response to an environmental stressor, producing a modified phenotype which may be either advantageous or deleterious. Then, when the stressor inducing this modified phenotype disappears, the organism takes a second novel developmental trajectory returning it to the pathway leading to the normal range of adult phenotypes that may well be more appropriate for the typical environmental condition (Figures 1B,C). Consider the following specific examples.

\section{Invertebrates}

Cold tolerance as adults can be acquired during cold exposure during early larval development in the fruit fly, Drosophila melanogaster. However, this modified, adaptive phenotype can be reversed during acclimation in the adult fly (Slotsbo et al., 2016). Interestingly, heat tolerance acquired during high temperature exposure as a fly larva is only partially reversible. This is reminiscent of Scenario B in Figure 1, where there can even be different degrees of reversibility for different phenotypic traits.

In the butterfly Bicyclus anynana, development temperature affects the size of the egg ultimately laid by the mature adults, with butterflies reared in cool temperatures producing larger eggs (Fischer et al., 2003). However, when the fully matured adults are switched to warmer temperatures for $\sim 10$ days, they will revert to the phenotype that lays smaller eggs, as if they had been reared as larvae in cooler temperatures. Thus, these temperature-induced phenotype modifications originating in larval development are quite reversible (Brakefield et al., 2007).

Developmental phenotypic switching of mouth formation has been examined in the model nematode Pristionchus pacificus (Werner et al., 2017). Different culture conditions (e.g., liquid vs. agar) during development "toggle" one of two distinctive differences in mouth form phenotype. Importantly, these effects are both immediate as well as reversible when culture medium is switched, indicating that the developmental trajectory of mouth formation can be adjusted by each set of gene-environment interactions resulting from culture conditions.

\section{Vertebrates}

Larval newts (Lissotriton boscai) at stage 45-47 change pigmentation as they develop in response to different ambient backgrounds, with light, high-reflecting environments inducing depigmentation and dark, low-reflecting environments resulting in enhanced pigmentation. Yet, pigment induction is completely reversible in adults depending upon backgrounds (Polo-Cavia and Gomez-Mestre, 2017).

Temperature-metabolism relations in the turtle Trachemys scripta elegans are strongly affected by temperature regimes during egg incubation (Ligon et al., 2012). However, the metabolic compensation to temperature, evident in hatchlings through both measurements of resting metabolic rate and growth rate, can be later reversed irrespective of life stage and irrespective of the embryonic developmental stage at which temperature stimuli were delivered.

Nutrient restriction during the first week of development in larval Xenopus laevis results in the failure of neural progenitors to proliferate, but this effect can be corrected by a return to normal nutrition, affected by a subsequent 10 -fold increase in cell proliferation stimulated by feeding (McKeown et al., 2017). Feeding also rescues nutrition restricted-induced decreases body length and brain tectal volume. Interestingly, the ability to rescue earlier developmental defects in the central nervous system induced by nutrient restriction during the CNS critical window (up to 7 days of development), does itself, have a critical window ( $\leq 9$ days).

Numerous examples exist of reversal of modified phenotypes at the cellular and molecular levels in vertebrates. Branching morphogenesis in the renal ureteric bud/collecting duct involve new branches sprouting from the tips of existing branches rather than from the stalks of the branches. However, when the tips are removed, the stalks can form new tips, indicating that the developmental transition from tip to stalk morphology during branching is reversible (Sweeney et al., 2008). Vascular smooth muscle cells show both extensive phenotypic plasticity and diversity during development. Environmental cues (e.g., mechanical stress, oxygen partial pressure) and the genes that respond to them result in phenotypic switching of smooth muscle cells when experiencing vascular injury, for example. This phenotype switching of both form and function during development is, however, fully reversible, likely involving epigenetic markers (Owens, 2007).

\section{Humans}

A well-documented and readily understood example of the effects of an adverse environment during critical windows for development is so-called 'catch-up' or compensatory growth in infants and children. Though these terms are frequently used interchangeably, a stricter definitional approach in the nonclinical literature uses "compensatory" to apply to an acceleration of growth rate, while "catch-up" more broadly allows for a return to conspecific control levels, which can be achieved by an extended period of growth with actual growth rate remaining at optimal levels (Arendt, 1997; Hector and Nakagawa, 2012). In humans, uterine nutritional insufficiency (an environmental stressor for the fetus) can result in abnormally low birth 


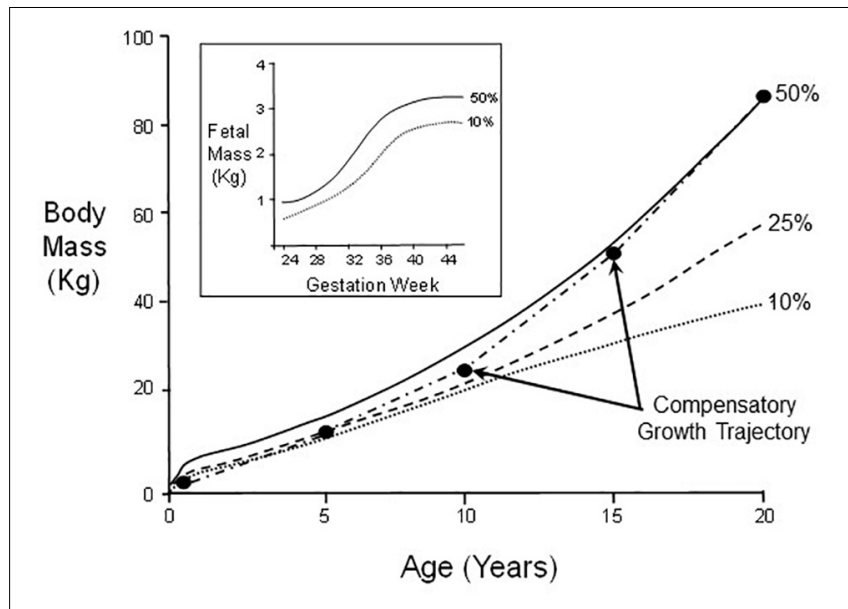

FIGURE 2 | Body mass changes in male humans between birth and age 20. Indicated are growth curves for 50th, 25th, and 10th percentiles of the population. A hypothetical example of catch-up growth is also indicated. Inset: Fetal mass as a function of gestation week for 50 th and 10 th percentiles.

weight. However, some low birth weight infants can exhibit compensatory growth, a post-natal period of greater than typical calorie acquisition and conversion to body mass at higher rates than a normally growing infant/child (Figure 2). Compensatory growth results in an atypical developmental trajectory that ultimately restores body mass to levels typical of its normal control cohort after a few years of early childhood (Cho and Suh, 2016; Martin et al., 2017). This is not "just" slowed growth, but rather an actual departure from normal range of phenotype for the infant's age, which is reversed (corrected) in later development.

Noteworthy is that while normal body mass per se is restored by adulthood (Scenario C in Figure 1), other general pathological phenotypic modifications resulting from nutrition restriction during critical windows for fetal development or early neonatal growth may not themselves be fully reversed (Scenario B in Figure 1). In the case of very low birth weight infants, this nutritional restriction during early development can lead to socalled "metabolic syndrome" in some adults, whereby increased risk exists for heart disease, stroke and type 2 diabetes (Martin et al., 2017). This underscores that any general phenotypic switching during development may simultaneously consist of both reversible and irreversible/pathological components (Figure 1). Consequently, experimenters need to be watchful for both categories of phenomena in experiments on developmental phenotypic switching, especially as there may be irreversible sub-components of a phenotype that are not revealed until development is complete.

Compensatory growth, and the related 'catch-up growth' during development, are not strictly a human or even mammalian phenomena. An extensive (but not exhaustive) meta-analysis identified these phenomena in 38 mammalian, 91 piscine, 39 avian and 30 arthropod species (Hector and Nakagawa, 2012). While not analyzed in this study, compensatory growth also occurs in amphibians and reptiles - e.g., Roark et al. (2009) and Burraco et al. (2019). Interestingly, the evolutionarily highly conserved nature of compensatory or catch-up growth suggests a high degree of biological significance. Yet, also important to note is that restoration of 'body mass' does not reflect appropriate restoration of all tissue types in proportion. In humans, the compensatory growth is often due to postnatal fat accumulation at the expense of muscle, in addition to the metabolic syndrome mentioned earlier (Okada et al., 2015). Thus, while body mass compensation may be obtained, the phenotype aberration caused by early undernutrition is not really fully corrected/reversed. Somewhat similarly, in lizards, regrowth of automized tails yields a structure with higher fat content than the original (Russell et al., 2015).

\section{"Truly Reversible" Phenotypic Switching During and After Development}

More extreme cases of true reversal involve an animal actually backtracking along the normal developmental trajectory. In the hydrozoans Turritopsis dohrnii (the 'immortal jellyfish') and Hydractinia carnea, for example, individuals follow one of several environment-dependent alternative developmental trajectories toward the adult medusa. Astonishingly, they can actually undergo an environmentally induced regression from the sexually mature medusa back into the polyp form when faced with stress in the form of starvation or elevated temperature or salinity. This regression can occur in as little as 3 days, increasing chances of survival in the face of environmental stress (Bavestrello et al., 2000; Schmich et al., 2007; Lisenkova et al., 2017).

Similar examples of such 'reversible acclimation' have been observed in the acorn barnacle, Balanus glandula. When adult barnacles reared in quiet water are transferred to elevated seawater flows, they can regain larval segments of their feeding legs lost during typical development (Kaji and Palmer, 2017).

Physiological reversal along developmental pathways has been documented in larvae and adults of the American bullfrog, Lithobates catesbeianus (Santin and Hartzler, 2016). Neuroventilatory responses to $\mathrm{CO}_{2}$ and $\mathrm{O}_{2}$ involved in modulating lung breathing were reduced in aquatic overwintering adult bullfrogs. More than just attenuated, however, the gas sensitivity profiles actually reverted to that of water larvae tadpoles.

Mature human osteoblasts subjected to simulated microgravity show altered pro-osteogenic determinants and a downregulation of bone differentiation marker and adhesive proteins. In a complete reversal of the normal developmental process, this pattern of cellular dedifferentiation allows reacquisition of migration potential by the primary osteoblasts (Gioia et al., 2018).

The examples of truly reversible development above bring into stark relief the many exceptions to the common viewpoint that modified phenotypes with their origins within developmental critical windows can, indeed, be reversible. Thus, a more comprehensive view of phenotypic switching enabled by developmental phenotypic plasticity is presented in 


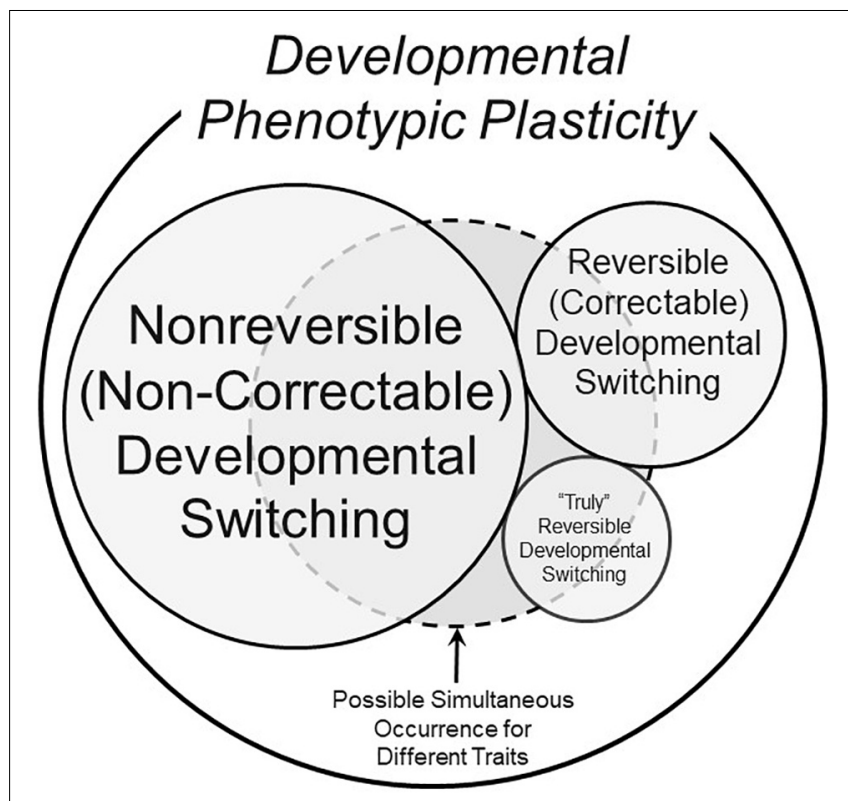

FIGURE 3 | A schematic of possible components of the overall phenomenon of developmental phenotypic plasticity. This Venn diagram makes the assumption that not all phenotypic changes induced by stressors during developmentally critical windows are non-reversible (non-correctable) - see text for further discussion of definitions. The different sizes of the circles and text are meant to convey the very approximate prevalence of the form of developmental switching. The dashed circle represents the fact that all three types of developmental phenotypic switching could occur simultaneously in an animal, each for a different trait.

Figure 3, which includes developmental phenotypic switching in three categories: traditional non-reversible (non-correctable), reversible (correctable) and "truly" reversible.

\section{Non-exclusivity of Types of Phenotype Switching}

Not all traits affected by development in a single animal necessarily follow the same pattern. As Lande (2014) indicates, "...many phenotypic characters of an individual develop and fluctuate continuously and reversibly throughout life in response to environmental change." Important to note is that, all too often (at least to this animal physiologist), "phenotype" refers to easily observed morphological features (e.g., size, color). In fact, an organism could for example be switching its physiology, biochemistry and behavior but not its gross external physiology. There may also be complex, subtle differences between developing phenotypic traits in terms of rates of change and metabolic associated costs (Lande, 2019). Likely most animals undergoing phenotypic switching in heterogeneous environments are 'mosaics', with some characters changing and others not, depending on the environmental cues.

Yet, as also emphasized above, we know that some characters do appear to become fixed during developmental critical windows. None of these scenarios are mutually exclusive. The domain circumscribed by the dashed line in Figure 3 indicates how, within a single animal, some traits could be non-reversible (non-correctable), others could be quite reversible (correctable), and still other traits might actually regress as an example of true reversibility.

As a conceptual example of multiple patterns of plasticity and subsequent phenotype switching within a single individual, consider an animal who's pulmonary morphometrics may be permanently altered during development because of hypoxic exposure during a critical window for lung development. However, the rate and depth at which they are ventilated at rest in the adult (physiology) could be a highly plastic physiological regulatory trait. As another example, a fish's musculature for swimming (morphology) could become fixed by experiences during development, but the characteristics of that fish's basic schooling (behavior) as an adult could be reset by changes in fish density - thus creating the phenotype switching 'mosaic' referred to above. Unfortunately, empirical evidence of such changes is scarce. We know that bullfrogs exposed to hypoxia at various stages in larval development and as adults show highly differential responses associated with the timing of the hypoxic exposure. Larvae show highly plastic morphological characters associated with gas exchange, but these characters become fixed in the adult and are no longer affected by hypoxia (Burggren and Mwalukoma, 1983). In contrast, the same hypoxic exposures cause little change in the red blood cell properties, including blood $\mathrm{P}_{50}$, of larvae, whereas these variables remain high plastic in the adults (Pinder and Burggren, 1983). In the quail (Coturnix coturnix) and the chicken (Gallus domesticus), hypoxia experienced during different periods of embryonic incubation induces phenotypic switching of some morphological, physiological and hematological characters, but not others (Dzialowski et al., 2002; Chan and Burggren, 2005; Burggren and Elmonoufy, 2017). Certainly, more experiments designed to reveal specific patterns of phenotypic switching are warranted. To reiterate a point made earlier, if we start looking for these various forms of developmental phenotypic switching, we are likely to find them.

\section{REVERSIBLE PHENOTYPE SWITCHING AS A BRIDGE DURING PERIODS OF UNPREDICTABLE CLIMATE CHANGE}

Having made a case for reversible (correctable) phenotype switching enabled by developmental phenotypic plasticity, what might we speculate to be the selection pressures leading to its evolution? Advantages to mature organisms of reversible phenotype switching, as well as its evolution, have received considerable attention - for an entry into the literature see Gabriel (2005), Gabriel (2006), Piersma and van Gils (2010), Berman (2016), Chen et al. (2016), Pfab et al. (2016), Lazaro et al. (2019), and Ratikainen and Kokko (2019). Yet, as Beaman et al. (2016) state, “...the evolution of reversible acclimation can no longer be viewed as independent from developmental processes." Indeed, the phenomenon of 'developmental bias' is increasingly becoming an important component of understanding evolutionary process (Uller et al., 2018; Parsons et al., 2019). Unfortunately, relatively few 
studies have explicitly collected experimental data to determine the adaptive advantages of reversible phenotype switching in developing, as opposed to mature, organisms. Such data, if available, could then be used to infer and model the evolution of reversible (correctable) plasticity. Yet, we can still ask the question "Why might such reversibility be important"?

Many common environmental stressors are short-term, variable in magnitude and non-predictable in occurrence - e.g., Burggren (2018, 2019). Thus, the development of alternative traits may be adaptive for one set of environmental conditions, but maladaptive for another set which may quickly follow, especially if either the switched phenotype of the environmental conditions are extreme (Chevin et al., 2010; Chevin and Hoffmann, 2017; Burggren, 2018; Pelster and Burggren, 2018). For example, consider the development of the gills in a developing freshwater fish that typically dwells in air saturated water. The ability to develop enlarged branchial surfaces could be life-saving should temporary aquatic hypoxia occur. However, once the environment returns to its normal condition of air saturation, retaining an enlarged gas exchange surface into adulthood, or even into just the next developmental stage, is likely to be metabolically very costly. This additional cost arises from maintaining osmoregulatory balance that is challenged by the inward flood of water across the larger gill surface area. The ability to 'shed' a trait or suite of traits by reversing (correcting) a switched phenotype arising during development is likely to be highly adaptive to the developing animal or its adult form. In contrast, fixed phenotypes at the end of the developmental period are more likely to result in phenotype-environment mis-matches (Gomulkiewicz and Kirkpatrick, 1992; Padilla and Adolph, 1996; Dukas, 1998; Snell-Rood, 2012). Thus, one could anticipate a strong selection pressure for a form of plasticity that allowed reversible phenotype switching in developing organisms, especially when environmental stressors cycle at shorter frequencies than the life span of the organism (Gabriel, 2005, 2006). Mathematical modeling has shown that reversible phenotype switching can be beneficial, even in rhythmically and slowly changing environments (Pfab et al., 2016).

Reversibility of developmental phenotypic switching may be an important mechanism of surviving climate change. Simply put, the ability to reverse short-term phenotypic changes during development may be key to a species surviving short-term climate changes, especially when extreme and stochastic. Figure 4 shows a hypothetical scenario of two different organisms,

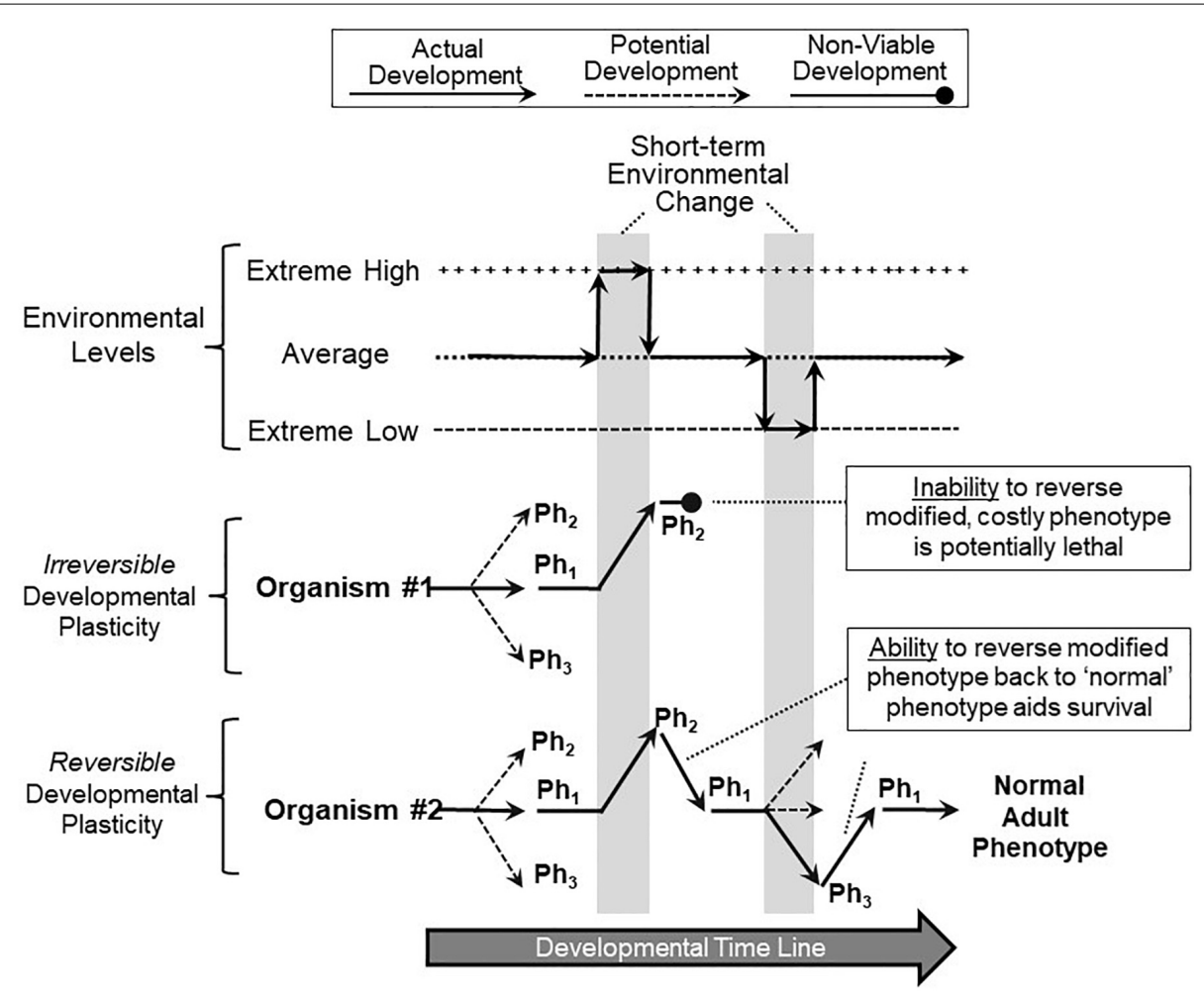

FIGURE 4 | Implications of reversible and non-reversible developmental phenotypic plasticity. In this scenario, two organisms are tracked during their development as they experience multiple, short-term extreme environmental changes (vertical gray bars). Both Organism \#1 (red) and Organism \#2 (blue) respond to the first extreme environmental change by switching from their normal range of phenotypes $\left(\mathrm{Ph}_{1}\right)$ to a specialized, adaptive phenotype $\left(\mathrm{Ph}_{2}\right)$ that allows continued survival in that environment. However, Organism \#1, lacking the ability to reverse (correct) its phenotype that was suitable for the extreme high environment, is now non-viable when the environment reverts back to more typical conditions as development continues. Organism \#2, which similarly responded to the first environmental change with a switched phenotype $\left(\mathrm{Ph}_{2}\right)$, has the ability to reverse or correct the costly or even lethal modified phenotype back to normal ranges $\left(\mathrm{Ph}_{1}\right)$ when the environment returns to normal, aiding its survival. Organism \#2 can also move to other phenotypes $\left(\mathrm{Ph}_{3}\right)$ and revert back to normal ranges $\left(\mathrm{Ph}_{1}\right)$ as subsequent environmental swings occur. Thus, in this overall scenario, reversible phenotypic switching during development heavily favors survival of the developing organism. 
both exhibiting developmental phenotypic plasticity, and both switching phenotype during development in response to the appearance of an adverse environment and its accompanying stressors. In this particular scenario, the short-term adverse environment then quickly and briefly returns to 'normal' (average) before actually changing to an adverse condition in the other direction. This might resemble extreme short-term weather events - for example, temperature swings - but could also mimic changes such as salinity or water availability. Let us further assume that the switched phenotype, essential for survival in a newly extreme environment, turns out to be costly, perhaps even lethal, for that organism when returned to a normally experienced or especially to a extreme low environment (as in the example of increased fish gill area, above). Under these circumstances, Organism \#1, which is unable to reverse a switched phenotype, becomes non-viable and may even fail to develop (Figure 4). In contrast, Organism \#2, capable of reversible phenotype switching, can revert to the original phenotype, continue development and live on to reproduce (Figure 4). Of course, there is a cost to all forms of plasticity (Snell-Rood, 2012; Lande, 2014; Pelster and Burggren, 2018), and not all phenotype switching is adaptive, especially in a stochastic environment (Padilla and Adolph, 1996; Burggren, 2018).

\section{COSTS AND BENEFITS OF REVERSIBLE PHENOTYPIC SWITCHING}

Having indicated that reversible phenotypic switching during development can indeed occur, a key question emerges: How costly is reversible phenotypic switching between different developmental pathways or trajectories? The answer hinges on the energetic/metabolic cost of developmental phenotypic switching between a modified phenotype and the normal range of phenotypes - and back. In a meta-analysis of phenotypic switching in 23 plant and animal species, the fitness costs of developmental plasticity were often found to be quite low (Van Buskirk and Steiner, 2009). Metabolic costs in particular of any magnitude can manifest themselves in terms of elevated metabolic levels or in the form of slowed growth. In fishes, physiological phenotype switching and reversal, occurring with acclimatization or laboratory acclimation, incurs variable degrees of metabolic costs (Jayasundara and Somero, 2013; Esbaugh et al., 2016). Phenotypic switching of behavioral phenotypes can be metabolically expensive given the high energetic cost of neural function and the additional neurons and neural connections that may be required (Hampson, 1991; Sporns et al., 2000; Snell-Rood, 2012; Karbowski, 2019). Changing morphological characters during development also has its costs, of course. For

\section{REFERENCES}

Ali, S., Champagne, D. L., Alia, A., and Richardson, M. K. (2011). Large-scale analysis of acute ethanol exposure in zebrafish development: a critical time window and resilience. PLoS One 6:e20037. doi: 10.1371/journal.pone.002 0037

Alvine, T., and Burggren, W. W. (2014). Renal, metabolic and hematological effects of trans-retinoic acid during critical developmental windows in the example, adaptive changes in head size in developing Australian tiger snakes (Notechis scutatus) resulted in slower rates of growth (Aubret and Shine, 2010). Morphological phenotype switching induced by predator cues in the developing freshwater crustacean Daphnia pulex had little apparent direct cost, but these may have been masked by an overall reduction in metabolic rate (Scheiner and Berrigan, 1998).

While in some instances the cost of a phenotypic switch and its reversal may not be prohibitively high, multiple cycles of switching caused by an environmental stressor followed by a reversal back to the normal range of phenotypes may be detrimental. Energetic reserves may be reduced or even exhausted, associated with oxidative stress being incurred. Developmental time and trajectories or even longevity even affected (Snell-Rood, 2012), to mention just a few negative consequences. Of course, the cost may pivot on the ability to acquire resources before, during and after each phenotype switching cycle.

\section{CONCLUSION}

Consideration of climate change in a developmental context becomes all the more useful when expanding the concept of developmental phenotypic switching to include switching that is reversible subsequent to the critical window. Appreciating the potentially reversible (correctable) nature of at least some traits evoked by abrupt, bidirectional environmental changes could prove to be key to understanding and predicting how individuals, populations and species will cope during climate change, especially when the changes are highly variable and unpredictable.

\section{AUTHOR CONTRIBUTIONS}

WB wrote this manuscript and constructed all figures.

\section{FUNDING}

This research was made possible by a grant (RECOVER 1 SA-1520) from The Gulf of Mexico Research Initiative (GoMRI).

\section{ACKNOWLEDGMENTS}

Dr. Ed Dzialowski and Mr. Christopher Melendez provided many useful comments during the development of this manuscript.

embryonic chicken. J. Comp. Physiol. B 184, 107-123. doi: 10.1007/s00360-0130777-9

Angiletta, M. J. J. (2009). Thermal Adaptation: A Theoretical and Empirical Synthesis. Oxford: Oxford University Press.

Arendt, J. D. (1997). Adaptive intrinsic growth rates: an invtegration across taxa. Q. Rev. Biol. 72, 149-177. doi: 10.1086/419764

Aristotle (350BC). On the Parts of Animals, trans. W. Ogle [The Internet Classics Archive]. Available at: http://classics.mit.edu//Aristotle/parts_animals.html 
Aubret, F., and Shine, R. (2010). Fitness costs may explain the post-colonisation erosion of phenotypic plasticity. J. Exp. Biol. 213, 735-739. doi: 10.1242/jeb. 040576

Bateson, P. (2017). Robustness and plasticity in development. Wiley Interdiscip. Rev. Cogn. Sci. 8, e1386.

Bateson, P., Gluckman, P., and Hanson, M. (2014). The biology of developmental plasticity and the Predictive Adaptive Response hypothesis. J. Physiol. 592, 2357-2368. doi: 10.1113/jphysiol.2014.271460

Bautista, N. M., and Burggren, W. W. (2019). Parental stressor exposure simultaneously conveys both adaptive and maladaptive larval phenotypes through epigenetic inheritance in the zebrafish (Danio rerio). J. Exp. Biol. 222:jeb208918. doi: 10.1242/jeb.208918

Bavestrello, G., Puce, S., Cerrano, C., and Sara, M. (2000). Phenotypic plasticity in hydrozoans: morph reversibility. Riv. Biol. 93, 283-294.

Beaman, J. E., White, C. R., and Seebacher, F. (2016). Evolution of plasticity: mechanistic link between development and reversible acclimation. Trends Ecol. Evol. 31, 237-249. doi: 10.1016/j.tree.2016.01.004

Berman, J. (2016). Ploidy plasticity: a rapid and reversible strategy for adaptation to stress. FEMS Yeast Res. 16:fow020. doi: 10.1093/femsyr/fow020

Brakefield, P. M., Pijpe, J., and Zwaan, B. J. (2007). Developmental plasticity and acclimation both contribute to adaptive responses to alternating seasons of plenty and of stress in Bicyclus butterflies. J. Biosci. 32, 465-475. doi: 10.1007/ s12038-007-0046-8

Burggren, W. (2018). Developmental phenotypic plasticity helps bridge stochastic weather events associated with climate change. J. Exp. Biol. 221:jeb161984. doi: $10.1242 /$ jeb. 161984

Burggren, W., and Mwalukoma, A. (1983). Respiration during chronic hypoxia and hyperoxia in larval and adult bullfrogs (Rana catesbeiana). I. Morphological responses of lungs, skin and gills. J. Exp. Biol. 105, 191-203.

Burggren, W. W. (2016). Epigenetic inheritance and its role in evolutionary biology: re-evaluation and new perspectives. Biology 5:24. doi: 10.3390/ biology5020024

Burggren, W. W. (2017). Epigenetics in insects: mechanisms, phenotypes and ecological and evolutionary implications. Adv. Insect Physiol. 53, 1-30. doi: 10.1016/bs.aiip.2017.04.001

Burggren, W. W. (2019). Inadequacy of typical physiological experimental protocols for investigating consequences of stochastic weather events emerging from global warming. Am. J. Physiol. Regul. Integr. Comp. Physiol. 316, R318R322. doi: 10.1152 /ajpregu.00307.2018

Burggren, W. W., Christoffels, V. M., Crossley, D. A. II, Enok, S., Farrell, A. P., Hedrick, M. S., et al. (2014). Comparative cardiovascular physiology: future trends, opportunities and challenges. Acta Physiol. 210, 257-276. doi: 10.1111/ apha. 12170

Burggren, W. W., and Crews, D. (2014). Epigenetics in comparative biology: why we should pay attention. Integr. Comp. Biol. 54, 7-20. doi: 10.1093/icb/icu013

Burggren, W. W., and Elmonoufy, N. A. (2017). Critical developmental windows for morphology and hematology revealed by intermittent and continuous hypoxic incubation in embryos of quail (Coturnix coturnix). PLoS One 12:e0183649. doi: 10.1371/journal.pone.0183649

Burggren, W. W., and Mueller, C. A. (2015). Developmental critical windows and sensitive periods as three-dimensional constructs in time and space. Physiol. Biochem Zool. 88, 91-102. doi: 10.1086/679906

Burggren, W. W., and Reyna, K. S. (2011). Developmental trajectories, critical windows and phenotypic alteration during cardio-respiratory development. Respir. Physiol. Neurobiol. 178, 13-21. doi: 10.1016/j.resp.2011. 05.001

Burraco, P., Valdes, A. E., and Orizaola, G. (2019). Metabolic costs of altered growth trajectories across life transitions in amphibians. J. Anim. Ecol. doi: 10.1111/1365-2656.13138 [Epub ahead of print].

Chan, T., and Burggren, W. (2005). Hypoxic incubation creates differential morphological effects during specific developmental critical windows in the embryo of the chicken (Gallus gallus). Respir. Physiol. Neurobiol. 145, 251-263. doi: 10.1016/j.resp.2004.09.005

Chen, X., Wang, Y., Feng, T., Yi, M., Zhang, X., and Zhou, D. (2016). The overshoot and phenotypic equilibrium in characterizing cancer dynamics of reversible phenotypic plasticity. J. Theor. Biol. 390, 40-49. doi: 10.1016/j.jtbi.2015. 11.008

Chevin, L. M., and Hoffmann, A. A. (2017). Evolution of phenotypic plasticity in extreme environments. Philos. Trans. R. Soc. Lond. B Biol. Sci. 19:372.
Chevin, L. M., Lande, R., and Mace, G. M. (2010). Adaptation, plasticity, and extinction in a changing environment: towards a predictive theory. PLOS Biol. 8:e1000357. doi: 10.1371/journal.pbio.1000357

Cho, W. K., and Suh, B. K. (2016). Catch-up growth and catch-up fat in children born small for gestational age. Korean J. Pediatr. 59, 1-7. doi: 10.3345/kjp.2016. 59.1.1

Cox, R. L., Calderon, de Anda, F., Mangoubi, T., and Yoshii, A. (2018). Multiple critical periods for rapamycin treatment to correct structural defects in Tsc1-suppressed brain. Front. Mol. Neurosci. 11:409. doi: 10.3389/fnmol.2018. 00409

Daskalakis, N. P., Bagot, R. C., Parker, K. J., Vinkers, C. H., and de Kloet, E. R. (2013). The three-hit concept of vulnerability and resilience: toward understanding adaptation to early-life adversity outcome. Psychoneuroendocrinology 38, 1858-1873. doi: 10.1016/j.psyneuen.2013.06.008

de Jong, M., and Leyser, O. (2012). Developmental plasticity in plants. Cold. Spring Harb. Symp. Quant. Biol. 77, 63-73. doi: 10.1101/sqb.2012.77.014720

Debat, V., and David, P. (2001). Mapping phenotypes: canalization, plasticity and developmental stability. Trends Ecol. Evol. 16, 551-561.

Dietert, R. R., Etzel, R. A., Chen, D., Halonen, M., Holladay, S. D., Jarabek, A. M., et al. (2000). Workshop to identify critical windows of exposure for children's health: immune and respiratory systems work group summary. Environ. Health Perspect. 108(Suppl. 3), 483-490. doi: 10.1289/ehp.00108s3483

Dobzhansky, T. (1973). Nothing in biology makes sense except in the light of evolution. Am. Biol. Teachnol. 35, 125-129. doi: 10.2307/4444260

Donelson, J. M., Salinas, S., Munday, P. L., and Shama, L. N. S. (2017). Transgenerational plasticity and climate change experiments: where do we go from here? Glob. Chang. Biol. 24, 13-34. doi: 10.1111/gcb.13903

Dubansky, B. (2018). "The interaction of environment and chronlogical and developmental time," in Development and Environment, ed. V. J. Inglezakis (Cham: Springer), 9-40.

Dukas, R. (1998). "Evolutionary ecology of learning," in Cognitive Ecology: the Evolutionary Ecology of Information Processing and Decision Making, Ed. R. Dukas (Chicago: University of Chicago Press), 129-174.

Dzialowski, E. M., von Plettenberg, D., Elmonoufy, N. A., and Burggren, W. W. (2002). Chronic hypoxia alters the physiological and morphological trajectories of developing chicken embryos. Comp. Biochem. Physiol. A 131, 713-724. doi: 10.1016/s1095-6433(02)00009-0

Eme, J., Mueller, C. A., Manzon, R. G., Somers, C. M., Boreham, D. R., and Wilson, J. Y. (2015). Critical windows in embryonic development: shifting incubation temperatures alter heart rate and oxygen consumption of Lake Whitefish (Coregonus clupeaformis) embryos and hatchlings. Comp. Biochem. Physiol. A Mol. Integr. Physiol. 179, 71-80. doi: 10.1016/j.cbpa.2014.09.005

Esbaugh, A. J., Ern, R., Nordi, W. M., and Johnson, A. S. (2016). Respiratory plasticity is insufficient to alleviate blood acid-base disturbances after acclimation to ocean acidification in the estuarine red drum, Sciaenops ocellatus. J. Comp. Physiol. B 186, 97-109. doi: 10.1007/s00360-015-0940-6

Ferner, K., and Mortola, J. P. (2009). Ventilatory response to hypoxia in chicken hatchlings: a developmental window of sensitivity to embryonic hypoxia. Respir. Physiol. Neurobiol. 165, 49-53. doi: 10.1016/j.resp.2008.10.004

Fischer, K., Eenhoorn, E., Bot, A. N., Brakefield, P. M., and Zwaan, B. J. (2003). Cooler butterflies lay larger eggs: developmental plasticity versus acclimation. Proc. Biol. Sci. 270, 2051-2056. doi: 10.1098/rspb.2003.2470

Frazier, K. S. (2017). Species differences in renal development and associated developmental nephrotoxicity. Birth Defects Res. 109, 1243-1256. doi: 10.1002/ bdr2.1088

Gabriel, W. (2005). How stress selects for reversible phenotypic plasticity. J. Evol. Biol. 18, 873-883. doi: 10.1111/j.1420-9101.2005.00959.x

Gabriel, W. (2006). Selective advantage of irreversible and reversible phenotypic plasticity. Arch. Hydrobiol. 167, 1-4. doi: 10.1073/pnas.1408589111

Gioia, M., Michaletti, A., Scimeca, M., Marini, M., Tarantino, U., Zolla, L., et al. (2018). Simulated microgravity induces a cellular regression of the mature phenotype in human primary osteoblasts. Cell Death Discov. 4:59. doi: 10.1038/ s41420-018-0055-4

Golovin, R. M., and Broadie, K. (2016). Developmental experience-dependent plasticity in the first synapse of the Drosophila olfactory circuit. J. Neurophysiol. 116, 2730-2738. doi: 10.1152/jn.00616.2016

Gomulkiewicz, R., and Kirkpatrick, M. (1992). Quantitative genetics and the evolution of reaction norms. Evol. Dev. 46, 390-411. doi: 10.1111/j.1558-5646. 1992.tb02047.x 
Grote, K., Hobler, C., Andrade, A. J., Grande, S. W., Gericke, C., Talsness, C. E., et al. (2009). Sex differences in effects on sexual development in rat offspring after pre- and postnatal exposure to triphenyltin chloride. Toxicology 260, 53-59. doi: $10.1016 /$ j.tox.2009.03.006

Halldorsdottir, T., and Binder, E. B. (2017). Gene x environment interactions: from molecular mechanisms to behavior. Annu. Rev. Psychol. 68, 215-241. doi: 10.1146/annurev-psych-010416-044053

Hampson, S. (1991). Generalization and specialization in artificial neural networks. Prog. Neurobiol. 37, 383-431. doi: 10.1016/0301-0082(91)90008-o

Hector, K. L., and Nakagawa, S. (2012). Quantitative analysis of compensatory and catch-up growth in diverse taxa. J. Anim. Ecol. 81, 583-593. doi: 10.1111/j.13652656.2011.01942.x

Hempel, C. G. (1945). Studies in the logic of confirmation (I.). Mind 54, 1-26. doi: $10.1093 / \mathrm{mind} /$ liv.213.1

Hensch, T. K., and Bilimoria, P. M. (2012). Re-opening windows: manipulating critical periods for brain development. Cerebrum 2012:11.

Hsu, H. H., Chiu, Y. H., Coull, B. A., Kloog, I., Schwartz, J., Lee, A., et al. (2015). Prenatal particulate air pollution and asthma onset in urban children. identifying sensitive windows and sex differences. Am. J. Respir. Crit. Care Med. 192, 1052-1059. doi: 10.1164/rccm.201504-06580C

Ingber, S. Z., and Pohl, H. R. (2016). Windows of sensitivity to toxic chemicals in the motor effects development. Regul. Toxicol. Pharmacol. 74, 93-104. doi: 10.1016/j.yrtph.2015.11.018

Jablonka, E. (2013). Epigenetic inheritance and plasticity: the responsive germline. Prog. Biophys. Mol. Biol. 111, 99-107. doi: 10.1016/j.pbiomolbio.2012.08.014

Jayasundara, N., and Somero, G. N. (2013). Physiological plasticity of cardiorespiratory function in a eurythermal marine teleost, the longjaw mudsucker, Gillichthys mirabilis. J. Exp. Biol. 216, 2111-2121. doi: 10.1242/jeb. 083873

Josephs, E. B. (2018). Determining the evolutionary forces shaping G x E. New Phytol. 219, 31-36. doi: 10.1111/nph.15103

Kaji, T., and Palmer, A. R. (2017). How reversible is development? Contrast between developmentally plastic gain and loss of segments in barnacle feeding legs. Evolution 71, 756-765. doi: 10.1111/evo.13152

Karbowski, J. (2019). Metabolic constraints on synaptic learning and memory. J. Neurophysiol. 122, 1473-1490. doi: 10.1152/jn.00092.2019

Korzybski, A. (1933). Science and Sanity: An Introduction to Non-Aristotelian Systems and General Semantics. Oxfod: International Non-Aristotelian Libray.

Kowalski, W. J., Dur, O., Wang, Y., Patrick, M. J., Tinney, J. P., Keller, B. B., et al. (2013). Critical transitions in early embryonic aortic arch patterning and hemodynamics. PLoS One 8:e60271. doi: 10.1371/journal.pone.0060271

Kunes, J., and Zicha, J. (2006). Developmental windows and environment as important factors in the expression of genetic information: a cardiovascular physiologist's view. Clin. Sci. 111, 295-305. doi: 10.1042/cs20050271

Lage, K., Greenway, S. C., Rosenfeld, J. A., Wakimoto, H., Gorham, J. M., Segre, A. V., et al. (2012). Genetic and environmental risk factors in congenital heart disease functionally converge in protein networks driving heart development. Proc. Natl. Acad. Sci. U.S.A. 109, 14035-14040. doi: 10.1073/pnas.1210730109

Lande, R. (2014). Evolution of phenotypic plasticity and environmental tolerance of a labile quantitative character in a fluctuating environment. J. Evol. Biol. 27, 866-875. doi: $10.1111 /$ jeb. 12360

Lande, R. (2019). Developmental integration and evolution of labile plasticity in a complex quantitative character in a multiperiodic environment. Proc. Natl. Acad. Sci. U.S.A. 116, 11361-11369. doi: 10.1073/pnas. 1900528116

Lazaro, J., Hertel, M., Muturi, M., and Dechmann, D. K. N. (2019). Seasonal reversible size changes in the braincase and mass of common shrews are flexibly modified by environmental conditions. Sci. Rep. 9:2489. doi: 10.1038/s41598019-38884- 1

Lenneberg, E. H. (1967). Biological Foundations of Language. New York, NY: John Wiley and Sons.

Ligon, D. B., Peterson, C. C., and Lovern, M. B. (2012). Acute and persistent effects of pre- and posthatching thermal environments on growth and metabolism in the red-eared slider turtle, Trachemys scripta elegans. J. Exp. Zool. A Ecol. Genet. Physiol. 317, 227-235. doi: 10.1002/jez.1716

Lisenkova, A. A., Grigorenko, A. P., Tyazhelova, T. V., Andreeva, T. V., Gusev, F. E., Manakhov, A. D., et al. (2017). Complete mitochondrial genome and evolutionary analysis of Turritopsis dohrnii, the "immortal" jellyfish with a reversible life-cycle. Mol. Phylogenet. Evol. 107, 232-238. doi: 10.1016/j.ympev. 2016.11.007
Lloyd, C. M., and Saglani, S. (2017). Development of allergic immunity in early life. Immunol. Rev. 278, 101-115. doi: 10.1111/imr.12562

Manuck, S. B., and McCaffery, J. M. (2014). Gene-environment interaction. Annu. Rev. Psychol. 65, 41-70. doi: 10.1146/annurev-psych-010213-115100

Martin, A., Connelly, A., Bland, R. M., and Reilly, J. J. (2017). Health impact of catch-up growth in low-birth weight infants: systematic review, evidence appraisal, and meta-analysis. Matern. Child Nutr. 13. doi: 10.1111/mcn.12297

Matesanz, S., Gianoli, E., and Valladares, F. (2010). Global change and the evolution of phenotypic plasticity in plants. Ann. N. Y. Acad. Sci. 1206, 35-55. doi: 10.1111/j.1749-6632.2010.05704.x

Matesanz, S., Horgan-Kobelski, T., and Sultan, S. E. (2012). Phenotypic plasticity and population differentiation in an ongoing species invasion. PLoS One 7:e44955. doi: 10.1371/journal.pone.0044955

McKeown, C. R., Thompson, C. K., and Cline, H. T. (2017). Reversible developmental stasis in response to nutrient availability in the Xenopus laevis central nervous system. J. Exp. Biol. 220, 358-368. doi: 10.1242/jeb.151043

McVey Neufeld, K. A., Luczynski, P., Seira Oriach, C., Dinan, T. G., and Cryan, J. F. (2016). What's bugging your teen?-The microbiota and adolescent mental health. Neurosci. Biobehav. Rev. 70, 300-312. doi: 10.1016/j.neubiorev.2016.06.005

Mendez-Sanchez, J. F., and Burggren, W. W. (2019). Hypoxia-Induced developmental plasticity of larval growth, gill and labyrinth organ morphometrics in two anabantoid fish: the facultative air-breather Siamese fighting fish (Betta splendens) and the obligate air-breather the blue gourami (Trichopodus trichopterus). J. Morphol. 280, 193-204. doi: 10.1002/jmor.20931

Moffitt, T. E., Caspi, A., and Rutter, M. (2005). Strategy for investigating interactions between measured genes and measured environments. Arch. Gen. Psychiatry 62, 473-481.

Mueller, C. A., Willis, C., and Burggren, W. W. (2016). Salt sensitivity of the morphometry of Artemia franciscana during development: a demonstration of 3-D critical windows. J. Exp. Biol. 219, 571-581. doi: 10.1242/jeb.125823

Munday, P. L., Warner, R. R., Monro, K., Pandolfi, J. M., and Marshall, D. J. (2013). Predicting evolutionary responses to climate change in the sea. Ecol. Lett. 16, 1488-1500. doi: 10.1111/ele.12185

Noh, S., Everman, E. R., Berger, C. M., and Morgan, T. J. (2017). Seasonal variation in basal and plastic cold tolerance: adaptation is influenced by both long- and short-term phenotypic plasticity. Ecol. Evol. 7, 5248-5257. doi: 10.1002/ece3. 3112

Okada, T., Takahashi, S., Nagano, N., Yoshikawa, K., Usukura, Y., and Hosono, S. (2015). Early postnatal alteration of body composition in preterm and small-for-gestational-age infants: implications of catch-up fat. Pediatr. Res. 77, 136-142. doi: 10.1038/pr.2014.164

Owens, G. K. (2007). Molecular control of vascular smooth muscle cell differentiation and phenotypic plasticity. Novartis Found. Symp. 283, 174-191.

Padilla, D. K., and Adolph, S. C. E. E. (1996). Plastic inducible morphologies are not always adaptive: the importance of time delays in a stochastic environment. Evol. Ecol. 10, 105-117. doi: 10.1007/bf01239351

Parsons, K. J., McWhinnie, K., Pilakouta, N., and Walker, L. (2019). Does phenotypic plasticity initiate developmental bias? Evol. Dev. 26:e12304.

Pelster, B., and Burggren, W. W. (2018). "Responses to environmental stressors in developing animals: costs and benefits of phenotypic plasticity," in Development and Environment, ed. V. J. Inglezakis (Cham: Springer), 97-113. doi: 10.1007/ 978-3-319-75935-7_5

Penfield, W., and Roberts, L. (1959). Speech and Brain Mechanisms. Princeton, NJ: Princeton University Press.

Pfab, F., Gabriel, W., and Utz, M. (2016). Reversible phenotypic plasticity with continuous adaptation. J. Math. Biol. 72, 435-466. doi: 10.1007/s00285-0150890-3

Piersma, T., and van Gils, J. (2010). The Flexible Phenotype: A Body-Centered Integration of Ecology, Physiology and Behaviour. Oxford: Oxford University Press.

Pigliucci, M., Schlichting, C. D., Jones, C. S., and Schwenk, K. (1996). Developmental reaction norms: the interactions among allometry, ontogeny and plasticity. Plant Species Biol. 11, 69-85. doi: 10.1111/j.1442-1984.1996. tb00110.x

Pinder, A., and Burggren, W. (1983). Respiration during chronic hypoxia and hyperoxia in larval and adult bullfrogs (Rana catesbeiana). II. Changes in respiratory properties of whole blood. J. Exp. Biol. 105, 205-213. 
Polo-Cavia, N., and Gomez-Mestre, I. (2017). Pigmentation plasticity enhances crypsis in larval newts: associated metabolic cost and background choice behaviour. Sci. Rep. 7:39739. doi: 10.1038/srep39739

Poore, K. R., Boullin, J. P., Cleal, J. K., Newman, J. P., Noakes, D. E., Hanson, M. A., et al. (2010). Sex- and age-specific effects of nutrition in early gestation and early postnatal life on hypothalamo-pituitary-adrenal axis and sympathoadrenal function in adult sheep. J. Physiol. 588, 2219-2237. doi: 10.1113/jphysiol.2010. 187682

Popper, K. (1959). The Logic of Scientific Discovery. New York, NY: Routledge.

Popper, K. (1970). "A Realist View of Logic, Physics and History," in Physics, Logic and History, Ed. W. Yourgrau (New York, NY: Plenum Press).

Quintaneiro, C., Soares, A., Costa, D., and Monteiro, M. S. (2019). Effects of PCB77 in adult zebrafish after exposure during early life stages. J. Environ. Sci. Health A Tox. Hazard Subst. Environ. Eng. 54, 478-483. doi: 10.1080/10934529. 2019.1568793

Ratikainen, I. I., and Kokko, H. (2019). The coevolution of lifespan and reversible plasticity. Nat. Commun. 10:538. doi: 10.1038/s41467-019-08502-9

Roark, A. M., Bjorndal, K. A., and Bolten, A. B. (2009). Compensatory responses to food restriction in juvenile green turtles (Chelonia mydas). Ecology 90, 2524-2534. doi: 10.1890/08-1835.1

Russell, A. P., Lynn, S. E., Powell, G. L., and Cottle, A. (2015). The regenerated tail of juvenile leopard geckos (Gekkota: Eublepharidae: Eublepharis macularius) preferentially stores more fat than the original. Zoology 118, 183-191. doi: 10.1016/j.zool.2014.12.003

Saltz, J. B., Bell, A. M., Flint, J., Gomulkiewicz, R., Hughes, K. A., and Keagy, J. (2018). Why does the magnitude of genotype-by-environment interaction vary? Ecol. Evol. 8, 6342-6353. doi: 10.1002/ece3.4128

Santin, J. M., and Hartzler, L. K. (2016). Environmentally induced return to juvenile-like chemosensitivity in the respiratory control system of adult bullfrog, Lithobates catesbeianus. J. Physiol. 594, 6349-6367. doi: 10.1113/ JP272777

Scheiner, S. M., and Berrigan, D. (1998). The genetics of phenotypic plasticity. VIII. The cost of plasticity in daphnia pulex. Evolution 52, 368-378. doi: 10.1111/j. 1558-5646.1998.tb01638.x

Schmich, J., Kraus, Y., De Vito, D., Graziussi, D., Boero, F., and Piraino, S. (2007). Induction of reverse development in two marine Hydrozoans. Int. J. Dev. Biol. 51, 45-56. doi: 10.1387/ijdb.062152js

Senner, N. R., Conklin, J. R., and Piersma, T. (2015). An ontogenetic perspective on individual differences. Proc. Biol. Sci. 07:282.

Skinner, M. K. (2011). Role of epigenetics in developmental biology and transgenerational inheritance. Birth Defects Res. C Embryo Today 93, 51-55. doi: $10.1002 /$ bdrc. 20199

Skinner, M. K. (2015). Environmental epigenetics and a unified theory of the molecular aspects of evolution: a Neo-Lamarckian concept that facilitates Neo-Darwinian evolution. Genome Biol. Evol. 7, 1296-1302. doi: 10.1093/gbe/ evv073

Slotsbo, S., Schou, M. F., Kristensen, T. N., Loeschcke, V., and Sorensen, J. G. (2016). Reversibility of developmental heat and cold plasticity is asymmetric and has long-lasting consequences for adult thermal tolerance. J. Exp. Biol. 219, 2726-2732. doi: $10.1242 / \mathrm{jeb} .143750$

Snell-Rood, E. C. (2012). Selective processes in development: implications for the costs and benefits of phenotypic plasticity. Integr. Comp. Biol. 52, 31-42. doi: $10.1093 / \mathrm{icb} / \mathrm{ics} 067$

Sommer, R. J., Dardiry, M., Lenuzzi, M., Namdeo, S., Renahan, T., Sieriebriennikov, B., et al. (2017). The genetics of phenotypic plasticity in nematode feeding structures. Open Biol. 7:160332. doi: 10.1098/rsob.160332

Spemann, H. (1938). Embryonic Development and Induction. New Haven, CT: Yale University Press.

Spicer, J. I., and Rundle, S. D. (2007). Plasticity in the timing of physiological development: physiological heterokairy-what is it, how frequent is it, and does it matter? Comp. Biochem. Physiol. A Mol. Integr. Physiol. 148, 712-719. doi: 10.1016/j.cbpa.2007.05.027

Sporns, O., Tononi, G., and Edelman, G. M. (2000). Connectivity and complexity: the relationship between neuroanatomy and brain dynamics. Neural Netw. 13, 909-922. doi: 10.1016/s0893-6080(00)00053-8

Standen, E. M., Du, T. Y., and Larsson, H. C. (2014). Developmental plasticity and the origin of tetrapods. Nature 513, 54-58. doi: 10.1038/nature 13708
Stockard, C. R. (1921). Developmental rate and structural expression: an experimental study of twins, 'double monsters' and single deformities, and the interaction among embryonic organs during their origin and development. Am. J. Anat. 28, 115-275.

Sweeney, D., Lindstrom, N., and Davies, J. A. (2008). Developmental plasticity and regenerative capacity in the renal ureteric bud/collecting duct system. Development 135, 2505-2510. doi: 10.1242/dev.022145

Tate, K. B., Kohl, Z. F., Eme, J., Rhen, T., and Crossley, D. A. II (2015). Critical windows of cardiovascular susceptibility to developmental hypoxia in common snapping turtle (Chelydra serpentina) embryos. Physiol. Biochem. Zool. 88, 103-115. doi: 10.1086/677683

Tills, O., Spicer, J. I., and Rundle, S. D. (2010). Salinity-induced heterokairy in an upper-estuarine population of the snail Radix balthica (Mollusca:Pulmonata). Aqua. Biol. 9, 95-105. doi: 10.3354/ab00231

Toth, Z., and Hettyey, A. (2018). Egg-laying environment modulates offspring responses to predation risk in an amphibian. J. Evol. Biol. 31, 710-721. doi: $10.1111 /$ jeb. 13258

Uller, T. (2008). Developmental plasticity and the evolution of parental effects. Trends Ecol. Evol. 23, 432-438. doi: 10.1016/j.tree.2008.04.005

Uller, T., Moczek, A. P., Watson, R. A., Brakefield, P. M., and Laland, K. N. (2018). Developmental bias and evolution: a regulatory network perspective. Genetics 209, 949-966. doi: 10.1534/genetics.118.300995

Utz, M., Jeschke, J. M., Loeschcke, V., and Gabriel, W. (2014). Phenotypic plasticity with instantaneous but delayed switches. J. Theor. Biol. 340, 60-72. doi: 10.1016/ j.jtbi.2013.08.038

Van Buskirk, J., and Steiner, U. K. (2009). The fitness costs of developmental canalization and plasticity. J. Evol. Biol. 22, 852-860. doi: 10.1111/j.1420-9101. 2009.01685.x

Vickers, M. H. (2011). Developmental programming of the metabolic syndrome critical windows for intervention. World J. Diabetes 2, 137-148. doi: 10.4239/ wjd.v2.19.137

Voss, P. (2013). Sensitive and critical periods in visual sensory deprivation. Front. Psychol. 4:664. doi: 10.3389/fpsyg.2013.00664

Wells, J. C. (2014). Adaptive variability in the duration of critical windows of plasticity: implications for the programming of obesity. Evol. Med. Public Health 2014, 109-121. doi: 10.1093/emph/eou019

Werner, M. S., Sieriebriennikov, B., Loschko, T., Namdeo, S., Lenuzzi, M., Dardiry, M., et al. (2017). Environmental influence on Pristionchus pacificus mouth form through different culture methods. Sci. Rep. 7:7207. doi: 10.1038/s41598-01707455-7

West-Eberhard, M. J. (2003). Developmental Plasticity and Evolution. Oxford: Oxford University Press.

West-Eberhard, M. J. (2005). Developmental plasticity and the origin of species differences. Proc. Natl. Acad. Sci. U.S.A. 102(Suppl. 1), 6543-6549. doi: 10.1073/ pnas. 0501844102

Wilson, R. S., and Franklin, C. E. (2002). Testing the beneficial acclimation hypothesis. Trends Ecol. Evol. 17, 66-70. doi: 10.1016/s0169-5347(01)02384-9

Woods, H. A. (2014). Mosaic physiology from developmental noise: withinorganism physiological diversity as an alternative to phenotypic plasticity and phenotypic flexibility. J. Exp. Biol. 217, 35-45. doi: 10.1242/jeb.089698

Zambonino-Infante, J. L., Mazurais, D., Dubuc, A., Queau, P., Vanderplancke, G., Servili, A., et al. (2017). An early life hypoxia event has a long-term impact on protein digestion and growth in juvenile European sea bass. J. Exp. Biol. 220, 1846-1851. doi: 10.1242/jeb.154922

Zhang, H., and Burggren, W. W. (2012). Hypoxic level and duration differentially affect embryonic organ system development of the chicken (Gallus gallus). Poult. Sci. 91, 3191-3201. doi: 10.3382/ps.2012-02449

Conflict of Interest: The author declares that the research was conducted in the absence of any commercial or financial relationships that could be construed as a potential conflict of interest.

Copyright (c) 2020 Burggren. This is an open-access article distributed under the terms of the Creative Commons Attribution License (CC BY). The use, distribution or reproduction in other forums is permitted, provided the original author(s) and the copyright owner(s) are credited and that the original publication in this journal is cited, in accordance with accepted academic practice. No use, distribution or reproduction is permitted which does not comply with these terms. 